\title{
\#HealthyKidsQuarantined: Supporting Schools and Families With Virtual Physical Activity, Physical Education, and Nutrition Education During the Coronavirus Pandemic
}

\author{
Laurel Whalen and Jeanne Barcelona \\ Wayne State University
}

\author{
Erin Centeio \\ University of Hawaii at Manoa
}

\author{
Nathan McCaughtry \\ Wayne State University
}

\begin{abstract}
When COVID-19 shuttered Michigan schools, 52 elementary and middle schools statewide were in various stages of implementation of comprehensive health programs, including the integration of physical activity, physical education, and nutrition education. To support the transition to a virtual learning environment, \#HealthyKidsQuarantined was launched, providing virtual physical activity, physical education, and nutrition education curriculum and resources. Content was distributed weekly via e-mail to teachers and families alongside a daily social media campaign that disseminated resources to a national audience. Results identified significant content usage by schools (21,300 views/downloads) and engagement through social media (9,800 views/downloads). Teachers, students, and families expressed value in the health content provided, stating it was a support needed in a time of chaos. This study suggests that providing virtual health content may be a feasible way to sustain school and family investment in comprehensive youth health. Furthermore, by utilizing multiple dissemination strategies, virtual programming may be an effective mechanism to expand reach.
\end{abstract}

Keywords: CSPAP, quarantine, COVID-19

On March 12, 2020, the World Health Organization announced that the outbreak of the coronavirus disease (COVID-19) had become a global pandemic (WHO, 2020). As cases of COVID19 began to rapidly spread throughout the United States, many state governments across the country determined that "Stay at Home" mandates were warranted to control the spread of the virus. As a result, schools across the country halted in-person learning in March and successively remained closed for in-person instruction for the balance of the school year.

Almost immediately upon closing buildings, schools shifted to a virtual learning format; however, this effort was fraught with challenges for teachers who largely lacked the resources, at-home technology, and pedagogical training necessary for such an undertaking. Previous research of planned, gradual shifts to online education illustrates the challenges and increased workload that teachers face related to using new technologies, communicating with students virtually, organizing synchronous learning, and assessing student work against educational metrics (Choi \& Park, 2006). However, these challenges were exacerbated by the abrupt shift to online learning best categorized as "emergency remote teaching" (ERT), defined as "a temporary shift of instructional delivery to an alternate delivery mode due to crisis circumstances" (Hodges, Moore, Lockee, Trust, \& Bond, 2020). In a survey of

Whalen, Barcelona, and McCaughtry are with the Department of Kinesiology, Health and Sports Studies, Wayne State University, Detroit, MI, USA. Centeio is with the Department of Kinesiology and Rehabilitation Science, University of Hawaii at Manoa, Honolulu, HI, USA. Whalen (Laurel.whalen@wayne.edu) is corresponding author. teachers between mid-March and early April 2020, Marshall, Shannon, and Love (2020) found that more than $90 \%$ of respondents had never taught online before the COVID-19 shift to ERT. In a March edition of Education Week, Milman (2020) suggested that educators had good reason to "pull back on their academic expectations" and encouraged them to establish curricular priorities upon which they should focus. Heeding this advice, many districts shifted their educational focus entirely toward core subjects, concentrating exclusively on reading, writing, and mathematics instruction. As a result, many schools struggled with other ways to meaningfully integrate noncore academic content, like physical education (PE), art, and music, into online learning. "In some cases, they [art, music, PE teachers] were asked not to assign any homework, so as to let students focus on their 'core' classes" (Marshall et al., 2020). Although schools have long been charged with fostering the development of the whole child, schools triaged their curricular efforts and built their capacity for remote instruction with little regard for PE or promoting physical activity (PA).

The challenges for teachers attempting to deliver online content were exacerbated by the sheer number of children across the country who lacked the resources to execute virtual learning at home. In March, nearly one-third of Michigan's school-aged children did not have Internet access or a home computer to engage in virtual learning (Higgins, 2020). Some districts worked to overcome this digital divide by creating paper curricular handouts for children. However, this delivery method primarily targeted core content areas, therefore further excluding "specials" classes, like PE. ERT was particularly difficult for PE teachers who struggled to include substantive content to support at-home PA often indoors in varying living spaces, with little to no equipment. Although some 
districts attempted to include live, virtual PE classes, many found it difficult to hold students accountable, which left PE teachers with no choice but to make the coursework optional. Even in cases where PE teachers were able to engage students in virtual curriculum, much of the onus of keeping children active and learning was placed on parents and caregivers. Across the country, more than 23 million working parents with children under the age of 14 had no available caregiver in the home (Bateman, 2020), thus presenting them with the challenge of simultaneously balancing working from home with supporting their children's education.

Physical education in schools has long been a critical mechanism for children to meet the recommended $60 \mathrm{~min}$ of daily PA (U.S. Department of Health and Human Services, 2008). Thus, the absence of it, coupled with the physical restrictions of home quarantine, presumably left many children with few opportunities to be active throughout the day. Compounding the lack of PE during at-home learning, the COVID-19 pandemic further restricted PA, with forced closures of recreational centers, public playgrounds, fitness facilities, and indoor and outdoor sports programs. As a result, there have been significant decreases in overall PA and increases in screen time and sedentary behavior for children in the United States (Bates et al., 2020). Despite being "in school" during home quarantine, the common reliance on asynchronous instruction resulted in a significant amount of unstructured time during traditional school hours. This is particularly troubling because research has established that other out-of-school time-like weekends and summer vacation-is more likely to promote sedentary behavior and unhealthy weight gain in children than time spent in school (Fairclough, Boddy, Mackintosh, Valencia-Peris, \& Ramirez-Rico, 2015; von Hippel \& Workman, 2016). Home quarantine and practicing social distancing also presents a significant risk of increased social isolation, which has been shown to "negatively impact mental health, PA patterns, and sedentary time across the lifespan" (Hall, Laddu, Phillips, Lavie, \& Arena, 2020). If the prolonged school closures and home quarantine result in similar inactivity patterns to other out-of-school time, there may be serious detrimental consequences for children's overall physical and mental health (Rundle, Park, Herbstman, Kinsey, \& Wang, 2020).

Schools have long been charged with fostering the development of the whole child, which has led to the promotion of inclusive approaches like the Comprehensive School Physical Activity Program (CSPAP), designed to integrate opportunities for health, promoting PA throughout the school day. Research has illustrated CSPAP's effectiveness in increasing PA and healthy eating, reducing obesity, and increasing academic achievement and attendance (Brusseau, Hannon, \& Burns, 2016; Centeio et al., 2014). Despite the critical importance of a child's home and community life in establishing a physically active lifestyle, research has suggested that "family and community involvement is one of the least frequently implemented components of CSPAP" (Cipriani, Richardson, \& Roberts, 2012). If family involvement and community engagement were important components of meeting the diverse needs of students in a traditional school setting (Griffin \& Farris, 2010), the pandemic has only intensified the significance of their role.

The COVID-19 school closures have, more than ever, forced teachers and school leaders to engage parents and caregivers as important stakeholders in meeting their educational objectives. Thus, the purpose of this study was to examine the perceptions of educators in providing virtual curriculum in $\mathrm{PA}, \mathrm{PE}$, and nutrition education during the COVID-19 pandemic. Furthermore, this study aims to investigate whether disseminating virtual wellness content is perceived by school leaders as a feasible way to promote and sustain school and family investment in comprehensive youth health initiatives.

\section{Methods}

When COVID-19 shuttered Michigan schools in March 2020, 52 elementary and middle schools statewide were in various stages of implementation of two different comprehensive wellness programs sponsored by The Center for Healthy and Community Impact at Wayne State University. The Dearborn School Health Through Integrated Nutrition and Education Strategies (DSHINES) program included programming in 17 elementary and middle schools in one suburban school district. The Building Healthy Communities (BHC) included 35 elementary schools from across the state of Michigan. Both programs focused on improving the wellness ideology within a school setting, emphasizing, in part, increased PA, quality PE, and integrating nutrition education into the curriculum. Prompted by the abrupt shift to ERT, the two intervention teams collaborated to continue delivering requisite program components and disseminating new, virtual lessons to teachers, students, and their families. To support the transition to virtual education, \#HealthyKidsQuarantined was launched with parallel objectives: disseminate curricular support to school personnel (principals, classroom teachers, and PE teachers) as they transitioned to online learning and provide resources for parents and caregivers to keep children healthy at home.

Over a 12-week period, from March 2020 through June 2020, daily PA and nutrition content was developed that was age appropriate and feasible to be implemented in a typical home setting. The PA lessons included resources for (a) promoting increased time in MVPA, including the recommended aerobic, muscular strength, and endurance activities; (b) skills-based activities aligned with the SHAPE national standards for PE (Society of Health and Physical Educators America, 2020); and (c) mindful activities focused on stress management and muscle relaxation. PA lessons utilized existing DSHINES and BHC program resources and also included curated content from a myriad of open-access sources, including SHAPE America, OPEN PE, GoNoodle, and Cosmic Kids Yoga. Lessons were organized to meet differing daily/weekly objectives (i.e., a gentle morning yoga stretch or an energizing dance for after lunch) and focused on providing $30 \mathrm{~min}$ of PA per day. In addition, United States Department of Agriculture (USDA)-aligned nutrition tips and activities were also provided, which combined nutrition knowledge with a healthy recipe, activity, or game. Nutrition lessons included topics such as the MyPlate model, the role of each food group, the importance of healthy beverages, and strategies for healthy snacking. PA and nutrition lessons were disseminated weekly as PDF documents with live links to online resources and were housed in each program's respective Google Drive.

The \#HealthyKidsQuarantined campaign included a concurrent, three-pronged approach to disseminating PA and nutrition content to maximize existing program structures and leverage relationships with respective program staff. In the DSHINES program, a weekly e-mail was sent to the district's school principals, PE coordinator, and PE teachers to alert them that new content was posted. The district's PE and health coordinator posted the content links on the district's PE blog, and PE teachers shared the links to the Google Drive folders with their students and families as part of their curricular initiatives or as additional enrichment resources. Principals shared the weekly e-mails directly with their classroom teachers so that they could also encourage students and 
families to engage in the content provided. The BHC program used two strategies to disseminate lessons to families learning at home: first, program staff distributed PA and nutrition content via a weekly e-mail to principals and teachers at participating schools, with the intent of them sharing it with students and families. Also, daily social media posts on BHC's Facebook, Twitter, and Instagram accounts included links to the lessons housed in the BHCspecific Google Drive. Finally, in an attempt to share the content with a wider audience, a third "public" Google Drive was created, and posts were shared on the social media channels of The Center for Healthy and Community Impact and via the personal networks of the Center's staff.

\section{Data Collection}

Data collection for the \#HealthyKidsQuarantined initiative was ongoing throughout the duration of the 12 weeks of lesson distribution. First, a weekly report generated from the respective program's social media accounts captured usage metrics, including the "likes," "shares," and click-throughs to access the lessons posted to Google Drive. To further support the data collection of the digital content usage, the program staff utilized Orangedox, an application that syncs with a cloud storage account (i.e., Google Drive) to create, in essence, a "virtual data room." Orangedox was ideal for this initiative for two reasons: (a) unlike direct sharing from Google Drive, with OrangeDox, recipients could preview and download any document or file hosted on Google Drive from any device, without needing a Google account; and (b) it offered detailed, page-by-page usage metrics. Throughout the 12-week initiative, Orangedox provided weekly audit logs with data on the activity within the drive, including document previews and downloads. These data were collected from each of the respective Google Drive folders (DSHINES, BHC, and public) used for the initiative.

At the conclusion of the academic year, data were also collected directly from the school users across 15 participating elementary and middle schools. Upon obtaining informed consent in accordance with Wayne State University's Institutional Review Board, an electronic survey was distributed to principals, classroom teachers, and PE teachers across one school district at the end of the 12-week distribution period. Given the limitations of COVID-19 and school closures, response rates were good for PE teachers $(96 \% ; n=24)$, moderate for principals $(40 \% ; n=6)$, but low for classroom teachers $(19 \% ; n=57)$. The survey instrument, initially drafted for program evaluation and altered for the COVID-19-modified virtual learning format, assessed how school closures impacted teachers' ability to incorporate healthy eating lessons and PA into student learning during ERT. Specifically, the survey included demographic items (i.e., age, gender, school, and years of experience); questions using Likert-scale ratings, to measure teachers' confidence with implementing health lessons and PA before and after school closures (e.g., "Over the course of this year, how confident were you in your ability to implement DSHINES healthy eating lessons in your classroom?" "How often did you encourage your students to engage in PA as part of their remote learning?"); and open-ended questions, to solicit data on their perceptions of the value of the virtual lesson content provided (e.g., "If you used DSHINES lessons, what did you find beneficial?").

\section{Data Analysis}

The survey instrument was previously used for program evaluation purposes, responses were initially deductively analyzed. The research team coded the data and organized the findings into initial themes. New data that emerged from the open-ended survey responses were subsequently inductively analyzed and categorized into new themes and subthemes. The research team met to confirm the thematic analysis, which informed the main interpretations of the study.

\section{Results}

\section{Descriptive User Statistics}

Descriptive user statistics were obtained from two sources: the Google analytics software attached to the social media accounts of BHC and the Center for Healthy and Community Impact and the Orangedox viewership metrics of the Google Drive folders for each respective program.

The DSHINES lessons were shared by program staff via e-mail and through district-initiated communication mechanisms (e-mail, PE coordinator's blog, and teachers' blog pages). The Orangedox viewership analytics reported 7,572 total document views and 1,535 downloads of the lessons from March 23 through June 12, 2020 ("views" and "downloads" were different in that documents could be read and content copied from the preview; however, the links within the document were only live if downloaded). The BHC program similarly distributed content links via e-mail to teachers and school staff at 35 participating schools. In addition, the BHC lessons were shared via the program's social media channels, accessed by 35 elementary schools actively programming during the 2019-2020 school year, as well as staff with schools who previously had the BHC program in their buildings. The Orangedox viewership metrics indicate 12,200 interactions with the online content from affiliated BHC schools, including 3,500 unique views and 2,500 content downloads during the 12-week school shutdown. As evidenced by the usage statistics, content dissemination in the district was easier to coordinate through the lead Health and PE teacher and among teachers already collaborating across the school district. Thus, the reach at the district level was far more significant than in the individual elementary buildings participating in the BHC.

Results of the third disseminations strategy, the \#HealthyKidsQuarantined campaign, indicated that social media engagement garnered 1,216 "likes" on Instagram, 1,684 reactions (comments/ shares) on Facebook, and 455 likes/retweets on Twitter. These daily social media posts linked users to the PA and nutrition lessons housed in a separate folder in Google Drive; resultingly, Orangedox analytics reported 12,361 page views were initiated from social media posts during the 12 -week period. The highest engagement occurred during the first 4 weeks of the campaign, with 6,685 views of the content during that time. The social media posts linked to documents hosted in the program's Google Drive - the link to which never changed. Thus, the drop-off in social media engagement could be explained by teachers accessing the content directly from Google Drive rather than initiating the engagement via social media.

\section{Qualitative Thematic Analysis}

Three predominant themes emerged from the findings: (a) school staff placed a high value on curricular support, (b) the virtual content was accessible for students at home, and (c) challenges to implementation were largely tied to overwhelmed family environments.

The first theme illustrated the value that school staff placed in receiving curricular support in a time of educational turmoil. 
Principals were especially supportive of the initiative, with $100 \%$ $(n=6)$ reporting that they both appreciated the support for their staff and promoted the virtual nutrition and PA lessons to their students and their families. While roughly $50 \%$ of classroom teachers $(n=27)$ integrated nutrition lessons and PA into their virtual classroom activities, more than $95 \%$ of the responding PE teachers $(n=22)$ utilized the provided lessons as their primary curricular content weekly during the school shutdown. PE teachers, in particular, appreciated that the curricular support that was "age-appropriate" (PE Teacher 13) and "nicely set-up and planned" (PE Teacher 20). Teachers felt that the virtual format largely negated their traditional teaching modalities (due to space limitations and lack of equipment for students learning at home) and forced educators to adjust their curricular goals from physical skill acquisition to the promotion of at-home PA. One PE teacher acknowledged this: "the links to physical activities were great to use with students ... I was thankful for the help" (PE Teacher 22).

The second theme illustrated the ease with which virtual content was disseminated to and utilized by students at home. The teachers in this study reported general challenges with adapting to technology, including a lack of a consistent platform to deliver content and establishing ways to track participation. However, teachers reported that the simple formatting (PDF and Word documents) of the curricular content provided by DSHINES made the content easily adaptable, and the open-access format fostered usability for students. The PA content in the lessons was largely curated from online sources, with links integrated into the lessons so they could be viewed even on small cell phone screens. One elementary PE teacher reiterated this ease of use: "the easy links to activities made it helpful even for younger students" (PE Teacher 6). Teachers reported that the format of the lessons was critical for optimal use, and including both structured activities (warm-up, moderate to vigorous PA, and cooldown) and options for varying intensity levels was important for usability. One teacher suggested that the lessons were useful because they were "easy to follow for the students" (PE Teacher 20). Another veteran PE teacher reiterated the value in providing options for athome PA as "students and their families could do as much or as little as they needed" (PE Teacher 1). Teachers reported that the curriculum was most effective when it encouraged students to "try a lot of new activities" (PE Teacher 3), "get outside more than normal" (PE Teacher 2), and "work on personal goals" (PE Teacher 12).

The third theme focused on overwhelmed family environments. Student access to technology notwithstanding, the predominant challenge for teachers in implementing the lessons was largely tied to stressful family environments. One classroom teacher described home learning as "chaotic" for many of her students (Classroom Teacher 4). Another classroom teacher suggested that although the lessons "were great at encouraging students to make room for physical activity at home and to make healthy choices," it was still challenging because accountability "falls on the parents more so than the teachers" (Classroom Teacher 18). Stressful at-home learning environments were especially difficult for PE teachers to manage, who reported that "parents were overwhelmed with classroom work," which created an environment where "physical education was not a priority" (PE Teacher 21). Many of the teachers' perceptions about the challenges of implementing virtual PA and nutrition lessons seemed symptomatic of the unique stressors of the pandemic (parents working remotely, home quarantine, unprepared schools and teachers, and lack of access to technology) and not indiscriminately with virtual learning.

\section{Discussion}

The COVID-19 pandemic forced teachers into ERT with virtually no training, an insufficient technology infrastructure, and limited curricular flexibility. Because of this, schools built their capacity for virtual instruction by largely prioritizing core subjects, leaving little room for integrations of "specials" classes like PE. Similarly, parents and caregivers at home were abruptly thrust into homeschooling, facing challenges with working from home, overseeing their children's educational activities, and accessing technology. The pandemic presented a uniquely chaotic moment in time for educators and families, unlikely to be replicated; however, it shed light on several important takeaways that suggest that providing virtual health and PA content may have promise as a feasible way to promote school and family investment in comprehensive youth health initiatives.

The findings of this study are initially compelling in the sheer number of users who accessed the material during the school closures. This suggests that the virtual nutrition and PA lessons created for the DSHINES and BHC programs filled significant gaps in the material, easily available for home use. There is not a dearth of wellness content for youth online; in fact, the opposite is true-a simple Internet search abounds with an overwhelming number of options. However, educators are then faced with the challenge of culling through the myriad of search results to find source material that is meaningful, age appropriate, and educational. Providing content that was vetted and intentionally planned served an important function for educators as they navigated the other concurrent challenges of ERT.

Online learning during the pandemic has also exacerbated youth screen time and sedentary behavior and limited the normal levels of active movement for school-aged children (Bates et al., 2020). While the pandemic has the potential for significant negative impacts on the health and well-being of children, it also underscores the need for a compulsory PE class as part of a supportive, healthy school environment. As schools shift back to in-person instruction, $\mathrm{PE}$ will continue to be a critical mechanism to foster the recommended 60 min of daily PA and teach students the importance of a healthy lifestyle. However, this study also suggests that virtual health and PA resources can be a productive complement to PE, promoting at-home PA and supporting families in making healthy choices. The use of multiple dissemination strategies and ensuring relevant, accessible content, virtual programming may prove to be an effective mechanism to further engage students and their families as part of a CSPAP model.

\section{Limitations}

This study is not without limitations. Given the unique circumstances of COVID-19 and the widespread need for support for educators, parents, and students, the research team determined that it was necessary to share resources without limitation; thus, there was no control group. As such, we were unable to collect data that would allow us to draw comparisons among those who did not participate in the initiative. Additionally, it was only feasible to administer the survey at one time point (end of the school year while in shut-down), which did not allow us to capture changes in educators' perceptions from the beginning of ERT to the end of the school year. This use of self-reported data and the fact that the variables during the 12-week lockdown were retrospectively assessed could have enhanced the risk of recall bias. In addition, this 
study did not consider the motivational variables, which could further contextualize teachers' engagement in promoting PA and healthy eating for their students during school closures. Finally, the high-stress environment of ERT and quarantine prevented our research team from following up on teachers' survey responses, which resulted in lower response rates than desired. The unique stressors of the pandemic also prevented our research team from collecting data directly from students and their caregivers, which could have provided important insights as to how best to support these stakeholders' engagement. Regardless of these limitations, these data captured novel and relevant information on ways that PA and PE can be fostered within a home setting, which will undoubtedly inform the research base.

\section{Conclusions}

Gobbi et al. (2020) suggested that the COVID-19 pandemic is "an opportunity to restore the role of PE in youths' lives," by meaningfully promoting PA as an extension of the home environment. The widespread use of PE, PA, and nutrition resources provided to educators, parents, and students during the COVID-19 quarantine via the \#HealthyKidsQuarantined campaign largely supports this notion. Results of the current study suggest not only widespread reach, but also a high value placed on the curricular support from educators and parents. Despite barriers to implementation during ERT, this study suggests that virtual PE/PA may be a viable mechanism to support CSPAP efforts to engage families and communities. Given that the COVID-19 pandemic is ongoing, and school closures remain widespread, future studies should aim to capture the perceptions of students and their caregivers helping to facilitate virtual PA, PE, and nutrition education. Continued research is also needed to examine the affordances of different online platforms employed by schools to identify optimal ways of using technology to support educators and parents in promoting meaningful engagement of healthy activities at home.

\section{Acknowledgments}

The authors thank Blue Cross Blue Shield of Michigan and the Michigan Health Endowment Fund for their support of the Building Healthy Communities and Dearborn SHINES programs, especially during the COVID-19 pandemic. This work was supported by grants from Blue Cross Blue Shield of Michigan, the Michigan Health Endowment Fund, the Michigan Department of Health and Human Services, and the Community Telecommunications Network.

\section{References}

Bateman, N. (2020, July 8). Working parents are key to COVID-19 recovery. Brookings. Retrieved from https://www.brookings.edu/ research/working-parents-are-key-to-covid-19-recovery/

Bates, L.C., Zieff, G., Stanford, K., Moore, J.B., Kerr, Z.Y., Hanson, E.D., ... Stoner, L. (2020). COVID-19 impact on behaviors across the 24-hour day in children and adolescents: Physical activity, sedentary behavior, and sleep. Children, 7(9), 138.

Brusseau, T., Hannon, J., \& Burns, R. (2016). The effect of a comprehensive school physical activity program on physical activity and healthrelated fitness in children from low-income families. Journal of Physical Activity and Health, 13, 888-894.

Centeio, E.E., McCaughtry, N., Gutuskey, L., Garn, A.C., Somers, C., Shen, B., ... Kulik, N.L. (2014). Physical activity change through comprehensive school physical activity programs in urban elementary schools. Journal of Teaching in Physical Education, 33(4), 573-591.

Choi, H.J., \& Park, J.H. (2006). Difficulties that a novice online instructor faced: A case study. Quarterly Review of Distance Education, 7(3), 317.

Cipriani, K., Richardson, C., \& Roberts, G. (2012). Family and community involvement in the comprehensive school physical activity program. Journal of Physical Education, Recreation \& Dance, 83(7), 20-26.

Fairclough, S.J., Boddy, L.M., Mackintosh, K.A., Valencia-Peris, A., \& Ramirez-Rico, E. (2015). Weekday and weekend sedentary time and physical activity in differentially active children. Journal of Science and Medicine in Sport, 18(4), 444-449.

Gobbi, E., Maltagliati, S., Sarrazin, P., di Fronso, S., Colangelo, A., Cheval, B., ... Carraro, A. (2020). Promoting physical activity during school closures imposed by the first wave of the COVID19 pandemic: Physical education teachers' behaviors in France, Italy and Turkey. International Journal of Environmental Research and Public Health, 17(24), 9431. PubMed ID: 33339228 doi:10.3390/ ijerph17249431

Griffin, D., \& Farris, A. (2010). School counselors and collaboration: Finding resources through community asset mapping. Professional School Counseling, 13(5), 248-256. doi:10.1177/2156759X1001300501

Hall, G., Laddu, D.R., Phillips, S.A., Lavie, C.J., \& Arena, R. (2020). A tale of two pandemics: How will COVID-19 and global trends in physical inactivity and sedentary behavior affect one another? Progress in Cardiovascular Diseases, 64, 108-110. doi:10.1016/j.pcad. 2020.04.005

Higgins, L. (2020, April 14). About 500,000 Michigan children lack the internet access and computers needed to learn at home. Chalkbeat Detroit. Retrieved from https://detroit.chalkbeat.org/2020/4/14/ 21225509/about-500-000-michigan-children-lack-the-internet-accessand-computers-needed-to-learn-at-home

Hodges, C., Moore, S., Lockee, B., Trust, T., \& Bond, A. (2020, March 27). The difference between emergency remote teaching and online learning. Educause Review. Retrieved from https://er.educause.edu/ articles/2020/3/the-difference-between-emergency-remote-teachingand-online-learning

Marshall, D., Shannon, D.M., \& Love, S.M. (2020). How teachers experienced the COVID-19 transition to remote instruction. Phi Delta Kappan, 103(2), 46-50.

Milman, N. (2020, March 30). This is emergency remote teaching, not just online teaching. Education Week. Retrieved from https://www. edweek.org/ew/articles/2020/03/30/this-is-emergency-remote-teachingnot-just.html

Rundle, A.G., Park, Y., Herbstman, J.B., Kinsey, E.W., \& Wang, Y.C. (2020). COVID-19-related school closings and risk of weight gain among children. Obesity, 28, 1008-1009.

Society of Health and Physical Educators America. (2020). National PE Standards: SHAPE Sets the Standard. Retrieved from https://www. shapeamerica.org/events/healthandphysicalliteracy.aspx

U.S. Department of Health and Human Services. (2008). Physical activity guidelines for Americans (Report). Washington, DC: Author.

von Hippel, P.T., \& Workman, J. (2016). From kindergarten through second grade, U.S. children's obesity prevalence grows only during summer vacations. Obesity, 24, 2296-2300.

WHO. (2020). WHO director-general's opening remarks at the mission briefing on COVID-19. Retrieved from https://www.who.int/dg/ speeches/detail/who-director-general-s-opening-remarks-at-themission-briefing-on-covid-19 